\title{
EMBOLIZACIÓN VERSUS CLIPAJE MICROQUIRÚRGICO EN ANEURISMAS CEREBRALES DE CIRCULACIÓN ANTERIOR
}

\author{
EMBOLIZATION VERSUS MICROSURGICAL CLIPPING IN CEREBRAL ANEURISMS ON \\ ANTERIOR CIRCULATION
}

\author{
EMBOLIZAÇ̃̃O VERSUS CLIPAGEM MICROCIRÚRGICA EM ANEURISMAS CEREBRAIS DE \\ CIRCULAÇÃO ANTERIOR
}

\author{
NATALIA BURGOS MORALES' ${ }^{1}$, PRISCILA SÁNCHEZ AGUIRRE², JORGE SARMIENTO BOBADILLA ${ }^{3}$, JIMMY ACHI ARTEAGA ${ }^{4,5}$, \\ DANIEL TETTAMANTI MIRANDA ${ }^{4,5,6}$ \\ 1 Hospital Universitario de Guayaquil, Guayaquil, Ecuador. \\ 2 Jackson Memorial Hospital, University of Miami, Florida, Estados Unidos. \\ ${ }^{3}$ Hospital Teodoro Maldonado Carbo, Guayaquil, Ecuador. \\ ${ }^{4}$ Grupo hospitalario Kennedy, Guayaquil, Ecuador. \\ ${ }^{5}$ Hospital Luis Vernaza, Guayaquil, Ecuador. \\ ${ }^{6}$ Universidad Católica de Santiago de Guayaquil, Guayaquil, Ecuador.
}

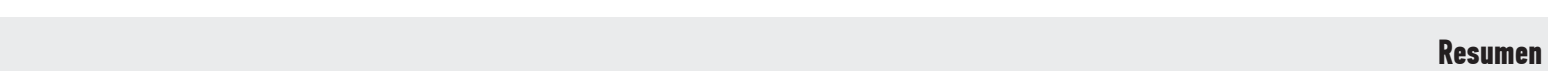

Objetivo: determinar el mejor procedimiento para el tratamiento de aneurismas cerebrales de circulación anterior, entre embolización y clipaje microquirúrgico, en términos de mortalidad y complicaciones. Materiales y métodos: estudio de cohorte, retroprospectivo de pacientes con hemorragia subaracnoidea por ruptura de aneurismas de circulación anterior; tratados mediante embolización o clipaje microquirúrgico en varios hospitales de Guayaquil, entre el 1 de enero de 2010 y el 31 de noviembre de 2011. Resultados: se incluyó 58 pacientes sometidos a embolización y 43 sometidos a clipaje. Se hallaron 113 aneurismas, localizados principalmente en la arteria comunicante posterior ( $39 \%, n=24)$. En lo que refiere a mortalidad, fallecieron $10 \%(n=6)$ de los pacientes embolizados y $14 \%$ ( $\mathrm{n}=6$ ) de los clipados; el riesgo relativo (RR) fue 0.71 , la reducción de riesgo relativo (RRR) fue 25,86 \% y la reducción absoluta del riesgo (RAR) fue 3,96 \% (IC95 $\%: 0.213-2.38, \mathrm{p}=0.57)$. Las complicaciones transoperatorias se presentaron en 3,54\% $(\mathrm{n}=4)$ del total de pacientes, siendo éstas la migración del dispositivo de embolización y la ruptura del aneurisma. Las complicaciones posoperatorias fueron infección, convulsionesy resangrado; presentándose en 6\% ( $\mathrm{n}=7$ ) de los casos. El RR para complicaciones fue 0.24, RRR=75,29\% y RAR= 15,76 \% (IC95 \%: 0.059-0.961, p=0.032), favoreciendo a los pacientes embolizados. Conclusión: la embolización presenta menor riesgo de complicaciones frente al clipaje de aneurismas de circulación anterior en pacientes con hemorragia subaracnoidea por ruptura aneurismática; sin embargo, no existen diferencias significativas en relación a mortalidad entre ambos procedimientos.
\end{abstract}

PALABRAS CLAVE: aneurisma intracraneal, hemorragia subaracnoidea, neurocirugía, embolización terapéutica.

Abstract

Objective: to determine the best procedure for treatment of cerebral aneurysms on anterior circulation, between embolization and microsurgical clipping, in terms of mortality and complications. Materials and methods: A retrospective cohort study of patients with subarachnoid hemorrhage from a rupture of anterior circulation aneurysms that had been treated by embolization or microsurgical clipping in several hospitals in Guayaquil between january 1, 2010 and november 31, 2011. Result: 58 patients who underwent embolization and 43 who underwent clipping were included. 113 aneurysms, located mainly in the posterior communicating artery $(39 \%, n=24)$ were found. Regarding mortality, $10 \%(n=6)$ of the embolized patients and $14 \%(n=6)$ of the clipped died. The relative risk (RR) was 0.71 , the relative risk reduction (RRR) was of $25.86 \%$ and the absolute risk reduction (ARR) was of $3.96 \%$ ( $95 \%$ Cl: $0.213-2.38, p=0.57$ ). Intraoperative complications occurred in $3.54 \%(n=4)$ of the total number of patients, consisting it in the migration of the embolization device and rupture of the aneurysm. Postoperative complications were infection, seizures, and rebleeding; present in $6 \%(n=7)$ of the cases. The RR for complications was $0.24, R R=75.29 \%$ and ARR $=15.76 \%$ (95\% Cl: 0.059-0.961, $p=0.032$ ), favoring embolized patients. Conclusion: embolization presents a lower risk of complications compared to clipping of anterior-circulation aneurysms in patients with subarachnoid hemorrhage from aneurysmal rupture; however, there are no significant differences in relation to mortality between the two procedures.

KEYWORDS: intracranial aneurysm, subarachnoid hemorrhage, neurosurgery, embolization, therapeutic.

Resumo

Objetivo: determinar o melhor método para o tratamento de aneurismas cerebrais da circulação anterior entre a embolização e clipagem microcirúrgica, em termos de mortalidade e complicações. Materiais e métodos: estudo de coorte, retroprospectivo de pacientes com hemorragia subaracnóide de aneurismas da circulação anterior rompidos; tratadas por embolização ou clipagem microcirúrgica em vários hospitais em Guayaquil, entre 1 de janeiro de 2010 e 31 novembro, 2011. Resultados: 58 pacientes submetidos a embolização e 43 submetidos a clipagem. Foram encontrados 113 aneurismas, localizados principalmente na artéria comunicante posterior $(39 \%, n=24)$. No que se refere mortalidade, $10 \%$ morreram $(n=6)$ dos pacientes embolizados e $14 \%(n=6)$ dos clipados; o risco relativo (RR) foi de 0,71 , a redução do risco relativo (RRR) foi $25,86 \%$ e a redução absoluta do risco (RRA) foi $3,96 \%$ (IC 95\%: 0,213-2,38, $p=0,57)$. As complicações transoperatorias ocorreron em 3,54\% $(n=4)$ de todos os pacientes, sendo estes a migração do dispositivo de embolização e a ruptura do aneurisma. Complicações pós-operatórias foram infecção, convulsões e novo sangramento; se apresentando em $6 \%$ ( $n=7$ ) dos casos. 0 risco relativo de complicações foi de 0,24, RRR=75,29\% e RAR= 15,76\% (IC 95\%: 0,059-0,961, $p=0,032$ ), favorecendo aos pacientes embolizados. Conclusão: A embolização tem um menor risco de complicações em comparação com a clipagem de aneurismas da circulação anterior em pacientes com aneurisma hemorragia subaracnóidea por ruptura, no entanto, não houve diferenças significativas em relação à mortalidade entre os dois procedimentos.

PALABRAS-CHAVE: aneurisma intracraniano, hemorragia subaracnóidea, neurocirurgia, embolização terapêutica. 
INTRODUCCIÓN

La hemorragia subaracnoidea (HSA) es responsable del 6 a $10 \%$ delos eventos cerebrovasculares, con una morbimortalidad superior a $90 \%$. Los aneurismas cerebrales son dilataciones anormales en la pared de las arterias responsables de aproximadamente $80 \%$ de las HSA no traumáticas. ${ }^{1-3}$ Los aneurismas localizados en la circulación anterior constituyen aproximadamente $90 \%$ de los aneurismas cerebrales; de ellos la mayoría se encuentra en la arteria comunicante anterior. ${ }^{1-5} \mathrm{El}$ tratamiento etiológico definitivo de la hemorragia subaracnoidea por ruptura aneurismática consiste en la colocación de clips metálicos en el cuello del aneurisma con técnica microquirúrgica, para su oclusión. En las últimas décadas se han implementado técnicas endovasculares usando "coils" para producir trombosis intraluminal del aneurisma. ${ }^{3,5-11}$

La terapia endovascular neurológica (TEN) ha sido aceptada como la terapia de elección en los aneurismas de circulación posterior, pero los aneurismas de circulación anterior presentan dificultades para dicho procedimiento, pues son lesiones que con frecuencia poseen cuellos anchos y anatomía compleja para la liberación de dispositivos intravasculares; en el caso de la arteria comunicante anterior, el origen de vasos hipotalámicos y quiasmáticos de diámetro muy pequeño, constituye un reto para la TEN. 5,10,11 En el Ecuador, la técnica endovascular tiene pocos años de desarrollo, pero se han observado buenos resultados inmediatos en pacientes sometidos a la misma. Sin embargo, no existe un estudio comparativo que permita mostrar resultados objetivos y deducir cuál es la terapia más beneficiosa.

El objetivo del presente estudio consiste en determinar el mejor procedimiento en el tratamiento de pacientes con HSA posterior a ruptura de aneurismas cerebrales de circulación anterior, entre embolización y clipaje microquirúrgico, en términos de mortalidad y complicaciones. La hipótesis propuesta es que la TEN presenta beneficio superior en el tratamiento de pacientes con HSA debida a ruptura de aneurismas cerebrales de circulación anterior, comparado con el clipaje microquirúrgico.

\section{MATERIALES Y MÉTODOS}

Estudio de cohorte, retroprospectivo, que incluyó todos los pacientes con HSA secundaria a ruptura de aneurismas cerebrales de circulación anterior; tratados mediante terapia endovascular o clipaje microquirúrgico en el Crupo Hospitalario Kennedy, Centro de Intervencionismo Angio Manabí, hospitales de la Policía Nacional de Guayaquil, Luis Vernaza y Naval de Guayaquil, desdeel 1 de enero de 2010 hasta el 31 de noviembre de 2011.

Los datos se obtuvieron bajo los códigos I-60 e I-72 del CIE-10 correspondientes a "HSA" y "otros aneurismas". Esta información se obtuvo de la base de datos de los centros antes mencionados previa solicitud escrita a los departamentos de estadística. Tomografías, angiografías y filmaciones, fueron facilitados por médicos de cada institución.

Los criterios de inclusión fueron: pacientes mayores de edad ( $\geq 18$ años), presencia de aneurisma roto que haya provocado HSA; aneurisma localizado en la circulación anterior: segmentos cavernoso y supraclinoideo de la arteria carótida interna y sus ramas (arteria oftálmica, arteria coroidea anterior, arteria comunicante posterior), arteria cerebral media, arteria cerebral anterior y arteria comunicante anterior. Los diagnosticados debieron realizarse por tomografía computarizada, angiotomografía o angiografía.

Los criterios de exclusión fueron: pacientes sometidos a ambos procedimientos (aquellos en los que se realizó embolización y luego clipaje) y en los que por las características del aneurisma debieron ser intervenidos por microcirugía, como pacientes con tortuosidad de vasos carotideos, estenosis carotidea y aquellos aneurismas de los que nacen ramas terminales, cuya embolización provocaría isquemia de territorios cerebrales.

Luego de seis meses, se realizó el seguimiento de los pacientes para evaluar estado neurológico, discapacidad física, muerte y control angiográfico. Posterior a recoger manualmente los datos en formularios se creó una base de datos en Microsoft Excel con tablas comparativas, que incluyeron las siguientes variables: edad, género, estadio clínico del paciente al ingreso según la Escala de Gravedad de Hemorragia Subaracnoidea de la World Federation of Neurosurgical Societies (WFNS), grado de HSA al ingreso según la Escala de Fisher, procedimiento realizado, complicaciones y valoración de muerte y discapacidad física posterior a la intervención según la Escala de Rankin Modificada. También se recolectaron los datos de la angiografía cerebral previa a la 
intervención, los cuales incluían: número de aneurismas por paciente, localización, tamaño, morfología, relación saco-cuello del aneurisma. El análisis estadístico de los datos se realizó mediante Microsoft Office Excel 2007 e IBM ${ }^{\circledR}$ SPSS ${ }^{\circledR}$ Statistics 20. La estadística descriptiva se realizó con frecuencia y porcentaje para variables cualitativas, mientras que para las variables cuantitativas se usó media y desviación estándar. En la estadística inferencial se midió el valor p con la prueba de contraste de hipótesis Chi Cuadrado de Pearson e intervalos de confianza $95 \%$. Se consideró un valor p estadísticamente significativo menor a 0.05 .

Para la realización de este estudio se siguieron las recomendaciones de la Declaración de Helsinki.

RESULTADOS

La Figura 1 muestra el flujo de pacientes durante el proceso de selección. El estudio incluyó 101 pacientes, de los cuales 58 fueron embolizados y 43 clipados. Los datos de género, edad e intervención realizada, así como las características de los aneurismas según tamaño, morfología y relación saco-cuello, se resumen en la Tabla 1.

\section{BÚSQUEDA INICIAL}

"HSA", "otros aneurismas"

Total: 152 pacientes.

- No tratados: 18

- Menos de 18 años: 3

- Circulación posterior: 11

Total: 32

Potenciales participantes: 120

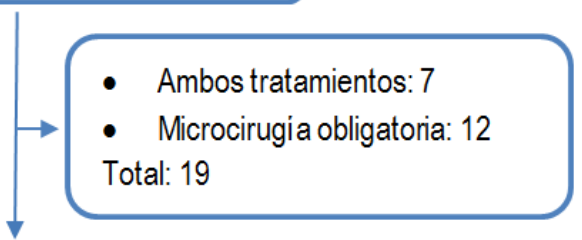

Seleccionados: 101

Figura 1. Flujo de selección de pacientes. HSA: hemorragia subaracnoidea. tabla 1. Características generales de la población ESTUDIADA * $†$

\begin{tabular}{|c|c|c|}
\hline CARACTERÍSTICAS GENERALES & $\begin{array}{r}\text { EMBOLIZACIÓN } \\
(N=58)\end{array}$ & $\begin{array}{r}\text { CLIPAJE } \\
(\mathrm{N}=\mathbf{4 3})\end{array}$ \\
\hline Número de aneurismas & 68 & 45 \\
\hline Edad (años) $\ddagger$ & $53,34 \pm 28,08$ & $47,44 \pm 26,60$ \\
\hline Masculino* & $21(36 \%)$ & $15(35 \%)$ \\
\hline Femenino* & $37(64 \%)$ & $28(65 \%)$ \\
\hline \multicolumn{3}{|l|}{ Número de aneurismas por paciente } \\
\hline $1^{*}$ & $46(79 \%)$ & $49(93 \%)$ \\
\hline $2^{*}$ & $10(17 \%)$ & $3(7 \%)$ \\
\hline $3^{*}$ & $1(2 \%)$ & $0(0 \%)$ \\
\hline $4^{*}$ & $1(2 \%)$ & $0(0 \%)$ \\
\hline \multicolumn{3}{|l|}{ Fisher § } \\
\hline$\left.\right|^{\star}$ & $21(36 \%)$ & $5(12 \%)$ \\
\hline$\|^{*}$ & $6(11 \%)$ & $15(35 \%)$ \\
\hline$\|\left.\right|^{*}$ & $17(29 \%)$ & $14(32 \%)$ \\
\hline IV* & $14(24 \%)$ & $9(21 \%)$ \\
\hline \multicolumn{3}{|l|}{ WFNS II } \\
\hline$\left.\right|^{\star}$ & $30(52 \%)$ & $13(30 \%)$ \\
\hline$\|^{*}$ & $11(19 \%)$ & $18(42 \%)$ \\
\hline$\| I^{*}$ & $11(19 \%)$ & $7(16 \%)$ \\
\hline $\mathrm{IV}^{*}$ & $4(7 \%)$ & $5(12 \%)$ \\
\hline$V^{*}$ & $2(3 \%)$ & $0(0 \%)$ \\
\hline \multicolumn{3}{|l|}{ Localización } \\
\hline Arteria carótida interna* & $4(6 \%)$ & $4(9 \%)$ \\
\hline Arteria oftálmica* & $8(12 \%)$ & $1(2 \%)$ \\
\hline Arteria coroidea anterior* & $2(3 \%)$ & $1(2 \%)$ \\
\hline Arteria comunicante posterior* & $23(34 \%)$ & $21(47 \%)$ \\
\hline Arteria cerebral media* & $16(23 \%)$ & $12(27 \%)$ \\
\hline Arteria comunicante anterior ${ }^{*}$ & $15(22 \%)$ & $6(13 \%)$ \\
\hline \multicolumn{3}{|l|}{ Tamaño } \\
\hline Pequeño $(<10 \mathrm{~mm} .)^{*}$ & $50(74 \%)$ & $40(89 \%)$ \\
\hline Grande $(10-25 \mathrm{~mm} .)^{*}$ & $15(22 \%)$ & $4(9 \%)$ \\
\hline Gigante $(>25 \mathrm{~mm} .)^{*}$ & $3(4 \%)$ & $1(2 \%)$ \\
\hline \multicolumn{3}{|l|}{ Morfología } \\
\hline Sacular* & $58(85 \%)$ & $45(100 \%)$ \\
\hline Fusiforme ${ }^{*}$ & $10(15 \%)$ & $0(0 \%)$ \\
\hline \multicolumn{3}{|l|}{ Relación saco/cuello } \\
\hline $1 \mathrm{a} 1^{*}$ & $27(40 \%)$ & $9(20 \%)$ \\
\hline $2 a_{1}^{*}$ & $30(44 \%)$ & $24(53 \%)$ \\
\hline $3 a 1^{*}$ & $11(16 \%)$ & $12(27 \%)$ \\
\hline \multicolumn{3}{|l|}{ Intervención } \\
\hline Embolización simple con coils* & $64(94 \%)$ & $0(0 \%)$ \\
\hline Embolización asistida por stent* & $1(2 \%)$ & $0(0 \%)$ \\
\hline Sole stenting ${ }^{*}$ & $3(4 \%)$ & $0(0 \%)$ \\
\hline Clipaje microquirúrgico* & $0(0 \%)$ & $45(100 \%)$ \\
\hline
\end{tabular}

* Se presenta el número de casos y entre paréntesis el porcentaje.

† Las variables: edad, género, número de aneurismas por paciente, grado WFNS, grado Fisher, fueron calculadas en relación al número de pacientes. Las variables: localización, tamaño, morfología, relación saco/cuello, intervención fueron calculadas en función del número de aneurismas.

‡ valor promedio más/menos dos desviaciones estándar.

§ Distribución de sangre en el espacio subaracnoideo según la escala de Fisher. Grado l: ausencia de sangre. Grado II: sangre difusa < $1 \mathrm{~mm}$. Grado III: sangre localizada >1mm. Grado IV: hemorragia intraparenquimatosa 0 intraventricular con 0 sin sangre difusa.

II Estadio clínico según la escala de la World Federation of Neurosurgical Societies. Grado I: GCS 15, ausencia de déficit motor. Grado II: GCS 13-14, ausencia de déficit motor. Grado III: GCS 13-14, déficit motor. Grado IV: GCS 7-12, ausencia o presencia de déficit motor. Grado V: GCS 3-6, ausencia 0 presencia de déficit motor. 
Se reportó complicaciones en $5 \%(n=3)$ de los pacientes embolizados y $19 \%(\mathrm{n}=8)$ de los clipados. Las complicaciones reportadas así como el porcentaje de presentación en cada grupo se muestran en la Figura 2. El RR para complicaciones en general fue 0.24 , con un intervalo de confianza $95 \%(0.059$ - 0.961), $\mathrm{p}=0.032$, RRR de $75,29 \%$ y RRA $15,76 \%$.

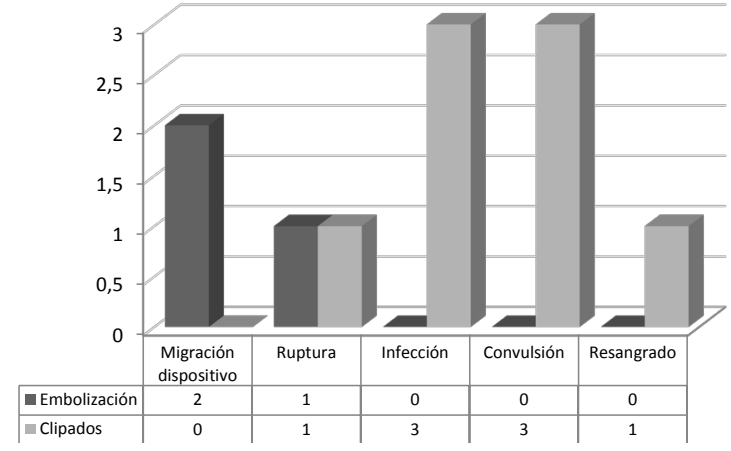

Figura 2. Complicaciones en pacientes embolizados y clipados. Fuente: departamento de estadística, hospital clínica Kennedy, centro de intervencionismo Angio Manabí, hospitales de la Policía Nacional de Guayaquil, Luis Vernaza y Naval de Guayaquil.

La evaluación posterior al tratamiento mediante la escala de Rankin modificada, muestra que $86 \%(n=51)$ y $72 \%(n=31)$, de los grupos embolizado y clipado respectivamente, permanecen en Rankin 1 (Figura 3).

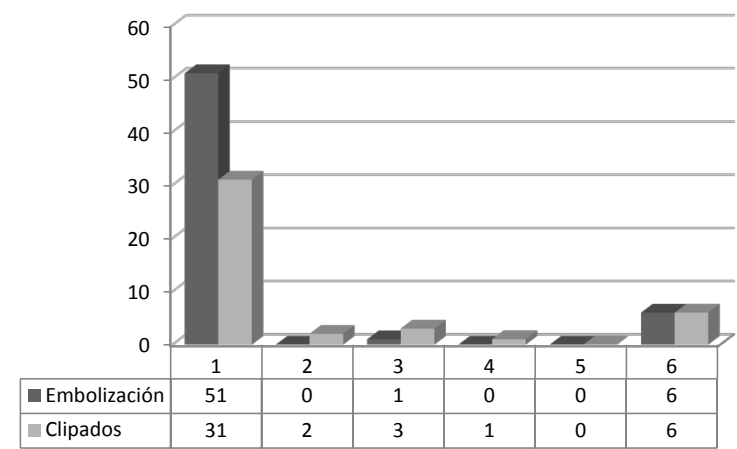

Figura 3. Grado Rankin de pacientes posterior a la intervención.

Fuente: departamento de estadística, hospital clínica Kennedy, centro de intervencionismo Angio Manabí, hospitales de la Policía Nacional de Guayaquil, Luis Vernaza y Naval de Guayaquil.

La mortalidad observada fue de $10 \%(\mathrm{n}=6)$ en los pacientes embolizados y $14 \%(n=6)$ en los pacientes clipados. La distribución de muerte en el postoperatorio inmediato (48 horas) y en el periodo tardío (3 días-6 meses), para cada grupo se encuentra en las Figuras 4 y 5. El RR de muerte encontrado fue 0.712 , con IC95\% $(0.213-2.38)$ y un valor p de 0.57 , RRR de $25,86 \%$, RRA de $0.036 \%$, a favor del tratamiento endovascular.

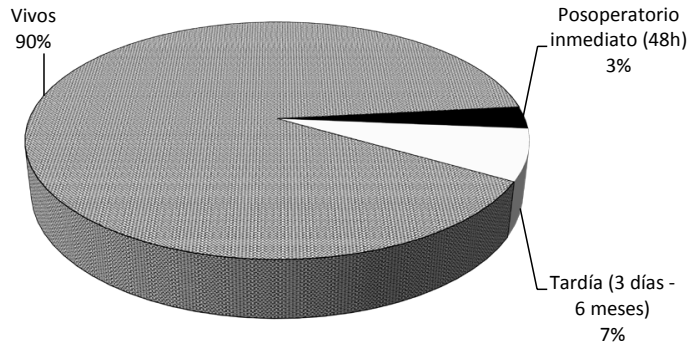

Figura 4. Mortalidad en pacientes embolizados.

Fuente: departamento de estadística, hospital clínica Kennedy, centro de intervencionismo Angio Manabí, hospitales de la Policía Nacional de Guayaquil, Luis Vernaza y Naval de Guayaquil.

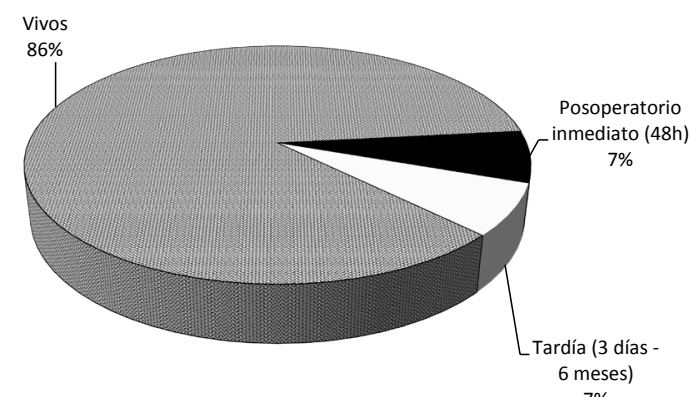

Figura 5. Mortalidad en pacientes clipados.

Fuente: departamento de estadística, hospital clínica Kennedy, centro de intervencionismo Angio Manabí, hospitales de la Policía Nacional de Guayaquil, Luis Vernaza y Naval de Guayaquil.

DISCUSIÓN

Los resultados obtenidos muestran que la intervención por terapia endovascular ofrece menos complicaciones, comparada con clipaje microquirúrgico.

Es conocido que la mayor parte de aneurismas de circulación anterior se ubican en la arteria comunicante anterior..$^{1-5}$ En los resultados obtenidos la localización predominante es la arteria comunicante posterior, ubicándose los aneurismas de arteria comunicante anterior en tercer lugar, tendencia que se mantiene en ambos grupos de intervención. La literatura indica que los aneurismas gigantes, con mala relación saco/cuello, fusiformes y de arteria cerebral media, no son buenos candidatos para tratamiento endovascular; sin embargo, el diseño de nuevos coils y técnicas como embolización asistida por balón o por stent y sole stenting; permiten obtener buenos resultados en los aneurismas mencionados. .,10-15 $^{-15}$ En este estudio se observó que todos los aneurismas gigantes, fusiformes, de arteria cerebral media, y $93 \%(n=25)$ de los aneurismas de cuello ancho se embolizaron sin complicación, realizando embolización asistida con stent en dos casos y sole stenting en uno. 
El ISAT, el estudio más reciente, amplio y randomizado que compara la embolización y el clipaje de aneurismas cerebrales, mostró en su informe inicial una RAR del $6.9 \%$ a favor del tratamiento endovascular, pero el seguimiento a cinco años no ha mostrado diferencia significativa en mortalidad y sobrevivencia sin discapacidad. Sin embargo, dicho estudio no incluyó pacientes en mal estado clínico ni aneurismas $>10 \mathrm{~mm}$. 5,16,17

En el presente estudio, a los seis meses, los indicadores relacionados con mortalidad (RR, RRR y RAR), mostraron resultados a favor del tratamiento endovascular; sin embargo, los valores de IC95 \% y el valor p muestran que los resultados no se deben atribuir a la intervención y pueden ser producto del azar. La relación entre mortalidad, estadio clínico y distribución de sangre en espacio subaracnoideo, permitió observar que más de la mitad de los pacientes fallecidos en el grupo embolizado, presentó mal estadio clínico (grados WFNS IV y V) y casi todos reportaban grado Fisher IV. En el grupo clipado, más de la mitad de los fallecidos presentaban buen estadio clínico (grados WFNS II y III); y la mitad se ubicaron en grado Fisher III. Se observa que en el grupo embolizado, la mayor parte de las muertes están relacionadas con mal estado clínico e imagenológico previo a la intervención; relación que no se cumple en los pacientes clipados.

Las complicaciones observadas fueron distintas en ambos grupos. En los embolizados, se presentaron complicaciones transoperatorias, que fueron: migración del dispositivo en aneurismas pequeños, saculares, con mala relación saco/cuello (1:1), lo que explica la salida del mismo al vaso padre; además, se presentó una ruptura de aneurisma durante la colocación del primer coil; sin embargo, se continuó con el procedimiento, logrando su exclusión de la circulación. Mientras que las complicaciones en los pacientes clipados se presentaron en el postoperatorio, correspondiendo a infección: dos casos de meningitis y una infección de colgajo quirúrgico. Estos eventos adversos pudieron relacionarse con la craneotomía realizada para el abordaje. Además se presentaron convulsiones y resangrado; siendo estas similares a las observadas en otros estudios. ${ }^{18,19,20}$ Un aneurisma presentó ruptura durante el abordaje, pero fue clipado con éxito.
Aproximadamente $88 \%$ de los pacientes de ambos grupos pertenecían a la clasificación I a III de la WFNS. Ningún paciente del grupo $\mathrm{V}$ de la WFNS fue sometido a craniectomía, probablemente por la tendencia de no someter pacientes en condición crítica al riesgo de una craniectomía si existe otro procedimiento disponible. Esta es una fuente posible de sesgo al comparar la mortalidad entre ambos procedimientos en pacientes con mal estado clínico.

Los resultados observados en relación a las complicaciones y los indicadores (RR, RRR y RAR), se mostraron a favor del tratamiento endovascular. Además, el intervalo de confianza y el valor de p demuestran que el menor riesgo de complicación en pacientes embolizados se debe a la intervención y no al azar.

La oclusión incompleta o la recanalización de los aneurismas posterior a la terapia endovascular se relaciona con resangrado en años posteriores; mientras que la oclusión completa y definitiva lograda con los clips microquirúrgicos es una de las principales ventajas teóricas de esta técnica. Una de las limitaciones de este estudio fue la imposibilidad de comparar el porcentaje de oclusión de aneurismas en ambos grupos, ya que el control angiográfico no es un procedimiento de rutina en el seguimiento de pacientes clipados en los hospitales en los que obtuvimos los datos.

\section{CONCLUSIÓN}

La embolización presenta menos riesgo de complicaciones que el clipaje microquirúrgico; a pesar que el RR reportado muestra una menor proporción de fallecimientos en pacientes sometidos a embolización comparado con aquellos a los que se realizó clipaje microquirúrgico. Sin embargo, los análisis estadísticos no muestran diferencias significativas en esta variable, por lo cual concluimos que no hay diferencia en la mortalidad de pacientes con HSA por ruptura de aneurismas de circulación anterior sometidos a embolización comparado con los que fueron clipados.

\section{REFERENCIAS BIBLIOGRÁFICAS}

1. Brown RD. Unruptured intracranial aneurysms. Semin Neurol. 2010;30(5):537-44.

2. Suárez J, Tarr R, Selman W. Aneurysmal Subarachnoid Hemorrhage. N Engl J Med. 2006;354:387-96. 
3. PreviglianoI. Hemorragia subaracnoidea decausa aneurismática. En: Basso A, Carrizo G, Mezzadri J. Neurocirugía aspectos clínicos y quirúrgicos. Rosario: Corpus; 2010. p. 490-503.

4. Vernooij MW, Ikram MA, Tanghe HL, Vincent AJ, Hofman A, Krestin GP. Incidental findings on brain MRI in the general population. N Engl J Med. 2007;357(18):1821-8.

5. Molyneux A, Kerr R, Stratton I, Sandercock P, Clarke M. International Subarachnoid Aneurysm Trial (ISAT) Collaborative Group. International Subarachnoid Aneurysm Trial (ISAT) of neurosurgical clipping versus endovascular coiling in 2143 patients with ruptured intracranial aneurysms: a randomised trial. Lancet. 2002;360(9342):1267-74.

6. Kelly M, Rasmussen P, Masaryk T. Intracranial aneurysms and subarachnoid hemorrhage. En: Masaryk T, Rasmussen P, Woo H. Endovascular Techniques in the Management of Cerebrovascular Disease. United Kingdom: Informa Healthcare; 2008. p. 111-32.

7. Quintana L, Pedroza A, Guzman A, Santos R. Guía de manejo de la hemorragia subaracnoidea aneurismática. En: Pedroza A, Quintana L, Perilla TA. Tratado de neurocirugía vascular latinoamericana. Bogotá: Legis; 2008. p. 97-111.

8. Raftopoulus C. Surgical management of intracranial aneurysms of the anterior circulation. In: Sindou M. Practical Handbook of Neurosurgery from Leading Neurosurgeons. New York: SpringerWien; 2009. p. 271-286.

9. Agrawal A, Sano H, Kato Y. Tratamiento quirúrgico de los aneurismas del circuito anterior. En: Basso AJ, Carrizo G, Mezzadri J. Neurocirugía aspectos clínicos y quirúrgicos. Rosario: Corpus; 2010. p. 520-526.

10. Zenteno M, Santos J, Aburto Y. Tratamiento endovascular en el manejo de los aneurismas intracraneales. En: Pedroza A, Quintana LP, Perilla TA. Tratado de neurocirugía vascular latinoamericana. Bogotá: Legis; 2008. p. 271-300.

11. Tjoumakaris S, Jabbour P, Veznedaroglu E. Tratamiento endovascular de los aneurismas del circuito anterior. En: Basso AJ, Carrizo G, MezzadriJ. Neurocirugía aspectos clínicos y quirúrgicos. Rosario: Corpus; 2010. p. 527-534.

12. Raja PV, Huang J, Germanwala AV, Gailloud P, Murphy KP, Tamargo RJ. Microsurgical clipping and endovascular coiling of intracranial aneurysms: a critical review of the literature. Neurosurgery. 2008;62(6):1187-202.
13. Lanzino G, Fraser K, Kankan Y, Wagenbach A. Treatment of ruptured intracranial aneurysms since the international Subarachnoid Aneurysm Trial: practice utilizing clip ligation and coil embolization as individual or complementary therapies. J Neurosurg. 2006;104(3):344-49.

14. Jeong SM, Kang SH, Lee NJ, Lim DJ. Stent-assisted coil embolization for the proximal middle cerebral artery fusiform aneurysm. J Korean Neurosurg Soc. 2010;47(5):406-8.

15. Bracard S, Abdel-Kerim A, Thuillier L, Klein O, Anxionnat R, Finitsis S, et al. Endovascular coil occlusion of 152 middle cerebral artery aneurysms: initial and midterm angiographic and clinical results. J Neurosurg. 2010;112:703-8.

16. Molyneux A, Kerr R, Birks J, Ramzi N, Yarnold J, Sneade M, etal. Risk of recurrent subarachnoid haemorrhage, death, or dependence and standardised mortality ratios after clipping or coiling of an intracranial aneurysm in the International Subarachnoid Aneurysm Trial. Lancet Neurol. 2009;8(5):427-33.

17. Bakker N, Metzemaekers J, Groen R, Mooi J, Van M. International Subarachnoid Aneurysm Trial 2009: Endovascular Coiling of Ruptured Intracranial Aneurysms Has No Signi cant Advantage Over Neurosurgical Clipping. Neurosurgery. 2010;66(5):961-62.

18. Molyneux A, Kerr R, Yu L, San Clarke M, Sneade M, Yarnold J, et al. International subarachnoid aneurysm trial (ISAT) of neu- rosurgical clipping versus endovascular coiling in 2143 patients with ruptured intracranialaneurysms: a randomised comparison of effects on survival, dependency, seizures, rebleeding, subgroups, and aneurysm occlusion. Lancet. 2005;366: 809-17.

19. Hoh B, Nothoo S, Chi Y, Mocco J, Barker F. Incidence of Seizures or Epilepsy After Clipping or Coiling of Ruptured and Unruptured Cerebral Aneurysms in the Nationwide Inpatient Sample Database: 2002-2007. Neurosurgery. 2011;69:644-50.

20. Connolly S, Hoh B, Selden N, Asher A, Kondziolka D, Boulis $\mathrm{N}$, et al. Clipping Versus Coiling for Ruptured Intracranial Aneurysms: Integrated Medical Learning at CNS 2007. Neurosurgery. 2010;66(1):19-34. 